\title{
Intramedullary spinal cord metastases: an increasingly common diagnosis
}

\author{
Sebastian Payer, MD, Klaus Christian Mende, Med Pract, Manfred Westphal, MD, and \\ Sven 0. Eicker, MD
}

Department of Neurosurgery, University Medical Center, Hamburg-Eppendorf, Germany

\begin{abstract}
OBJECT Intramedullary spinal cord metastases (ISCM) represent a small proportion of intramedullary tumors. However, with the lifespans of patients with malignant tumors increasing, incidents of ISCM are on the rise. Due to threateningly severe disabilities in patients, accompanied by limited life expectancy, every attempt should be made to treat these tumors the same way as metastases elsewhere in the CNS, with the goal of complete removal of the ISCM and preservation of neurological functions. The object of this study is to retrospectively analyze the experiences of 22 patients who were surgically treated for ISCM over a 22-year period.
\end{abstract}

METHODS Hospital charts of 22 patients, who were surgically treated for ISCM between 1992 and 2014, were reviewed retrospectively. Demographic data, histopathological diagnoses of primary cancer, chronological sequence of the disease, and neurological status using the simplified McCormick functional classification were collected and reanalyzed.

RESULTS The most frequent histology was metastasis of lung cancer, followed by brain and breast cancers. The time span from primary cancer diagnosis to the development of symptomatic spinal metastases ranged from 0 to 285 months, with a mean interval of 38 months. The leading presenting sign was dysesthesia ( $77 \%$ of the population), followed by paresis (68\%). Only 5 patients (23\%) showed urinary retention. Initial performance status represented by the McCormick Scale was on average 2.47. Total or near-total removal was achieved in $87 \%$ of cases. Compared with the clinical status 1-2 days after surgery, there was an improvement in the McCormick Scale grade at the last follow-up from 2.47 to 2.12 $(p=0.009)$. Likewise, an improvement was detected when comparing the preoperative status with the last follow-up (from 2.45 to $2.12 ; p=0.029$ ). The mean survival time after surgery was 11.6 months.

CONCLUSIONS These results suggest that surgery for intramedullary metastases-with all of the challenges of a rare and potentially risky procedure - can be beneficial to patients with advanced stages of cancer. Surgery can be performed with minimal new morbidity and results in maintaining neurological performance status.

http://thejns.org/doi/abs/10.3171/2015.5.FOCUS15149

KEY WORDS intramedullary metastases; spinal cord; intramedullary spinal cord metastases

$\mathrm{D}$ EVELOPMENT of intramedullary spinal cord metastases (ISCM) is still a rare event in the course of malignant diseases. However, it is on the rise due to more effective therapies that result in the prolongation of survival. ${ }^{8}$ This is reflected in the volume of recently published case reports and small series of ISCM. . $20,22,23,29,30,42,46$ Autopsy studies demonstrated an incidence of ISCM up to $2 \%$. Few ISCM are symptomatic and therefore not diagnosed.$^{51}$ Because of the low frequency of treated cases, therapy standards are still to be defined.
The main goal in treating ISCM is to maintain mobility and neurological functions for patients with limited life expectancy. Surgery is favored but is still second to primarily anti-edematous systemic therapy combined with or followed by radiation. However, the clinical benefit hereby achieved is often very limited and at the level of palliation is associated with considerable side effects, particularly from continuous steroid application. ${ }^{4,17}$ Reports of surgical removal of ISCM have become more frequent, however, with a wide range of neurological results. ${ }^{3-5,45}$ In 397 pa-

ABBREVIATIONS CUP = cancer of unknown primary; CUSA = Cavitron ultrasonic surgical aspirator; IOM = intraoperative monitoring; ISCM = intramedullary spinal cord metastases.

SUBMITTED March 26, 2015. ACCEPTED May 14, 2015.

INCLUDE WHEN CITING DOI: 10.3171/2015.5.FOCUS15149.

DISCLOSURE The authors report no conflict of interest concerning the materials or methods used in this study or the findings specified in this paper. 
tients with intramedullary lesions who underwent surgical treatment, a strategy of maximal removal was pursued. Of these 397 patients, the clinical findings in our cohort of 22 patients will be reviewed.

\section{Methods}

This study is a retrospective analysis of data from 22 patients who were surgically treated for ISCM. Hospital data collected over a period of 22 years (1992-2014), both at the time of treatment and at follow-up where available, were used. Six patients were treated before 2005, and 16 between 2006 and 2014, 15 of them within the last 4 years of the range, indicating a trend of higher incidence. The mean age of the patients was 55 years. No pediatric patients were included. Imaging was based on MRI in T1and T2-weighted images with and without gadolinium in sagittal, axial, and coronal slices. Histological examinations were performed in-house with standard histopathological and immunohistological staining. Data obtained from the hospital archives included demographics, histopathological diagnoses of primary cancer, site of primary cancer, clinical symptoms leading to the diagnosis of ISCM, time since the primary cancer was diagnosed, pre- and postoperative neurological deficit using the simplified McCormick Scale functional classification, ${ }^{41}$ location of the spinal metastasis, approximate radiological size of the metastasis, methods used during surgery, extent of resection, further treatments postoperatively, and ambulatory status at last follow-up. The extent of resection was based on the judgment of the surgeon and the postoperative MRI.

The McCormick Scale (simplified) is a functional classification of intramedullary spinal cord tumors with the appropriate validation. This was used to evaluate the clinical condition of the patients pre- and postoperatively, as well as in the follow-up (Table 1). ${ }^{41}$ Data are shown as the mean. Descriptive statistics were used to characterize the study population. The Student t-test was used to compare means of metric variables.

\section{Results}

\section{Demographic Data}

Since 1986, 397 intramedullary lesions have been treated in our institution, but metastases have been part of the spectrum only since 1992 . Since then, 22 cases have been treated, with the frequency markedly increasing during the last 4 years of this analysis.

Thirteen male patients and 9 female patients were treated (Tables 2 and 3). The mean age among all patients was 55 years (range 21-86 years). On average, the male population was younger than the female population $(\mathrm{p}=$ 0.48 ). The mean age of men was 52 years (range $21-86$ years). The mean age of women was 58 years (range 33-79 years). Metastases in the cervical and thoracic spine were identified in $77 \%$ of patients $(n=17)$, whereas metastases in the thoracolumbar region (i.e., conus medullaris) could be identified only in $23 \%$ of patients $(n=5)$. The timespan from primary cancer diagnosis to the development of symptomatic spinal metastases ranged from 0 to 285
TABLE 1. McCormick Scale functional classification of intramedullary spinal cord tumors

\begin{tabular}{cc}
\hline Grade & Characteristics \\
\hline I & Neurologically normal, mild focal deficits, normal gait \\
\hline II & $\begin{array}{c}\text { Sensorimotor deficits affecting function, severe pain, gait diffi- } \\
\text { culties, can still walk }\end{array}$ \\
\hline III & $\begin{array}{c}\text { Moderate neurological deficit, needs cane for ambulation, } \pm \\
\text { arms affected, } \pm \text { independent }\end{array}$ \\
\hline IV & As above + arms affected, usually not independent \\
\hline
\end{tabular}

months, with a mean interval of 38 months. In women, the period of time from primary cancer diagnosis to spinal metastasis was an average of 55.8 months. The corresponding period of time in men was remarkably shorter, with an average of 25.5 months $(p=0.35)$.

In 17 cases, the primary cancer was known or could be identified after obtaining the histopathology of the spinal metastasis. Lung cancer was the most frequent primary malignant disease (5 cases), followed by brain and breast cancers (3 cases each). Malignant melanoma was found twice. Bladder, prostate, ovarian, and kidney cancers, each represented by 1 case, were rare events. The brain metastases originated from 1 each anaplastic astrocytoma, ependymoma, and medulloblastoma.

In 8 patients, spinal metastases were the first clinical manifestation of malignant disease, thus representing a cancer of unknown primary (CUP) syndrome. In 5 of these patients, there was still no finding of a primary malignant tumor in subsequent staging. In 1 patient, the origin of the primary tumor was still unknown 12 months after treatment of ISCM. Another patient, in whom a renal carcinoma was the most probable origin of malignancy, died 4 months after spinal surgery without confirmation of the primary tumor source. Eleven patients (50\%) had additional metastases at the time of presentation, 9 of them (41\%) with tumors that had metastasized into the brain, 4 into the bone and liver, and 2 into the lung and lymph nodes. One patient had a skin metastasis (melanoma).

Fifty-nine percent of patients $(n=13)$ showed only mild clinical symptoms on the day of admission and were still able to walk autonomously (McCormick Scale Grade I or II). Eighteen percent of patients $(n=4)$ were severely disabled, with McCormick Scale Grade IV. Dysesthesia was the most common clinical symptom, with $77 \%$ of the study population reporting it before the time of surgery. The second-most common clinical symptom was paresis (68\% of patients). Only 5 patients (23\%) showed urinary and/or anal disorders.

\section{Findings on MRI}

In each case, the radiological finding was obtained by an MRI with and without gadolinium enhancement. T1 and T2 imaging sequences were obtained. The typical enhancement was an intramedullary nodulus, which could be seen in all but 1 case (Figs. 1 and 2). In this 1 case, which proved to be a metastasis of an anaplastic astrocytoma WHO Grade III, there was no enhancement in the gadolinium-enhanced T1 sequence, but the tumor could be clearly seen in the T2 FLAIR sequence (Fig. 3). 


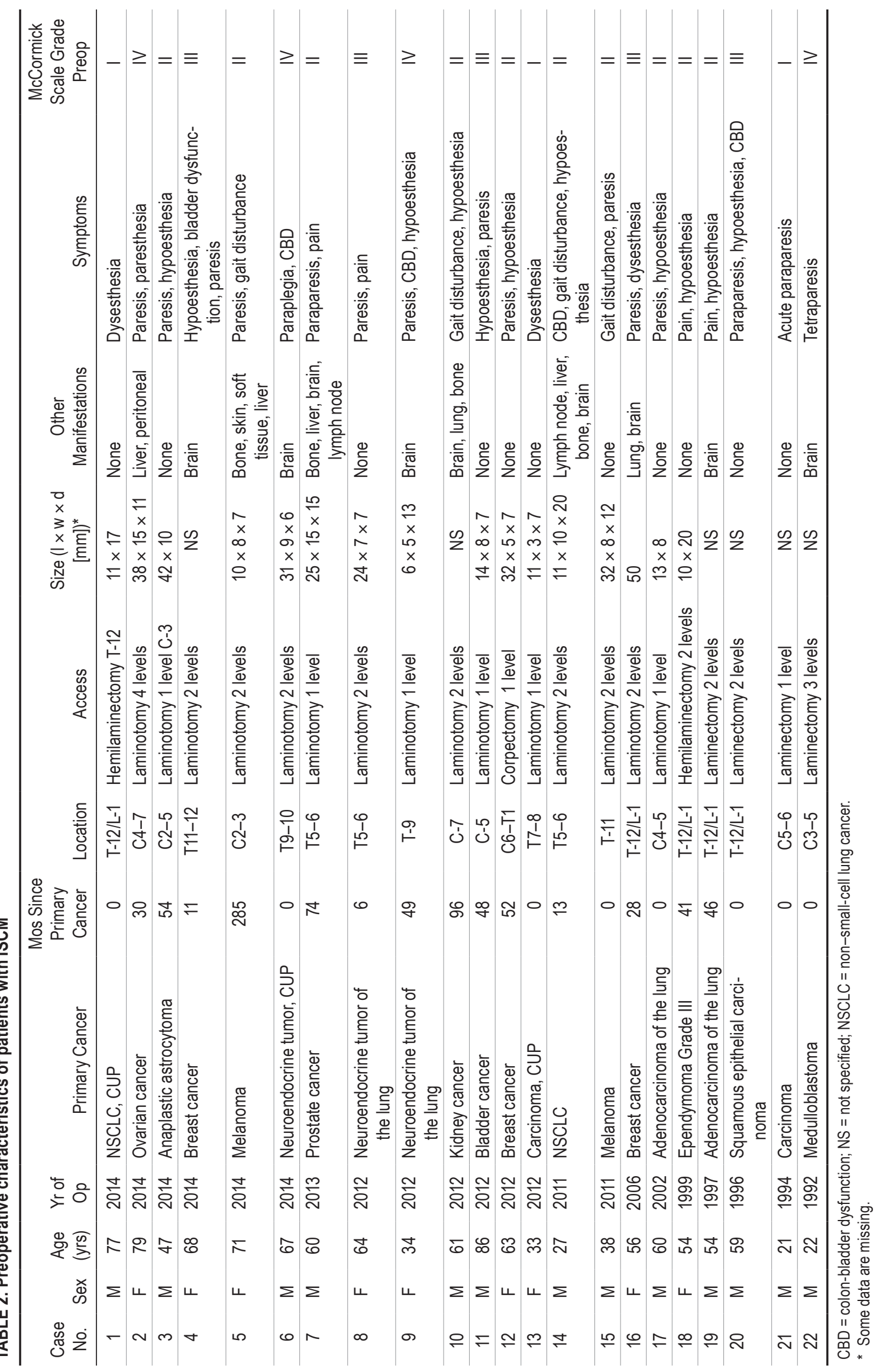




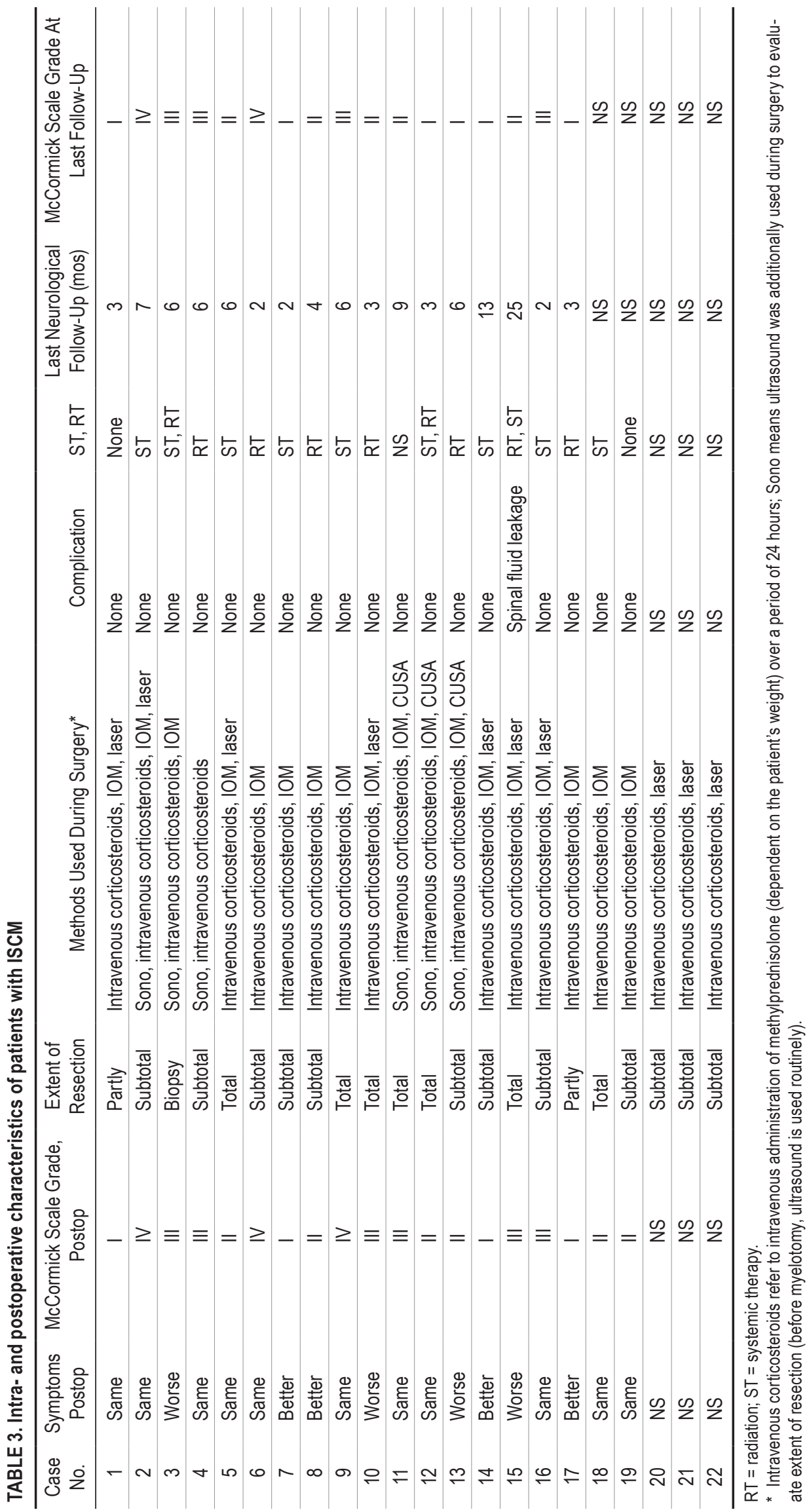




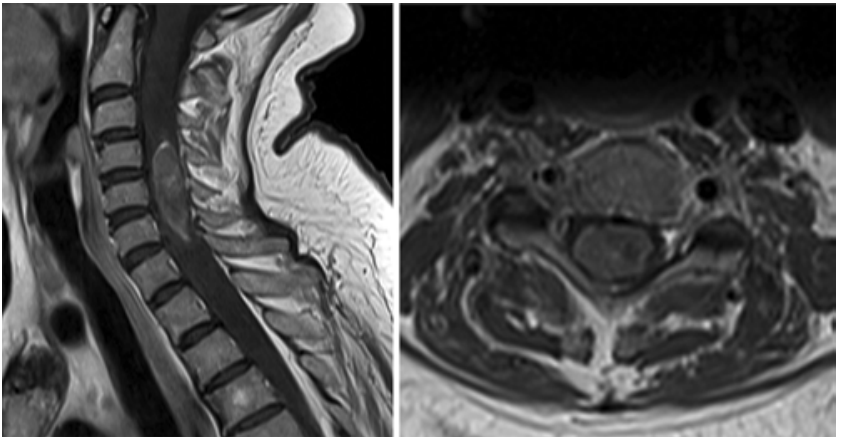

FIG. 1. Left: Typical enhancement in the gadolinium-enhanced T1 sequence of an intramedullary metastasis originating from an ovarian carcinoma at the level of C4-6. Right: The tumor extending in a transversal slice is shown.

The assumable volume of the tumor, where length (l), width (w), and depth (d) could be obtained, was calculated using the equation for ellipsoid volumes: vs $=v=4 / 3 \pi *$ $l * w * d$. Fifteen tumors could be calculated with a mean volume of $2980 \mu \mathrm{l}$, ranging from 513 to $8360 \mu \mathrm{l}$. In the remaining 7 patients, only 2 values could be established due to lack of original MRI data. Therefore, a volume could not be calculated. However, volume did not correlate with length of survival, neurological symptoms, extent of resection, or origin of the tumor.

\section{Therapy}

All 22 patients underwent microsurgical treatment, with 1 biopsy and 21 resections. In 7 patients (32\%), a total resection was obtained. In 12 patients (55\%), the final degree of resection could be described as subtotal (small layers on the border zone to the healthy spinal cord were left intentionally due to electrophysiological changes), and in 2 patients the tumor was only partly removed. In $77 \%$ of patients, a hemi-laminectomy or an osteoplastic laminotomy with reconstruction of the posterior spinal column was conducted to reach the intradural pathology. In the remaining 5 patients, a corpectomy $(\mathrm{n}=1)$ or laminectomy $(\mathrm{n}=4)$ was performed. Only in 2 patients $(9 \%)$ was $>2$ levels of lamina opening necessary to obtain the whole dimension of the tumor. After opening the dura, the spinal cord was approached through the sulcus medianus posterior, proving to be the approach with the lowest chance of neurological side effects.

Ultrasound was used in all cases to identify the dimension of the tumor prior to resection, especially in cases where the tumor did not reach the dorsal surface and therefore could not be identified visually through the mi-
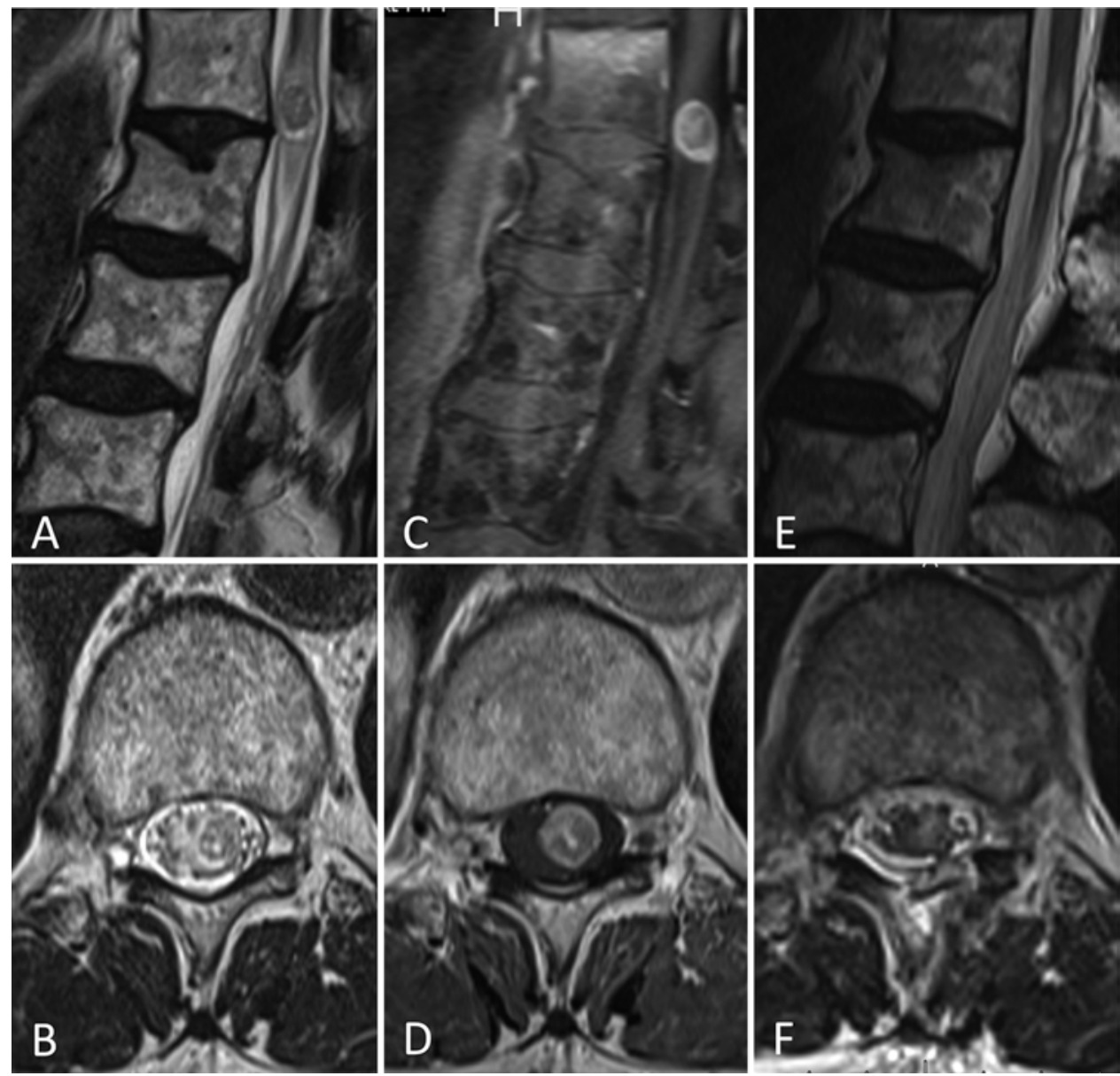

FIG. 2. Patient 1 with a space-occupying lesion in the conus medullaris in sagittal ( $A$ and $C$ ) and transversal (B and D) T2 and T1 sequences with contrast enhancement. The postsurgical results with remaining edema $(E)$ and the small approach $(F)$ are shown. 


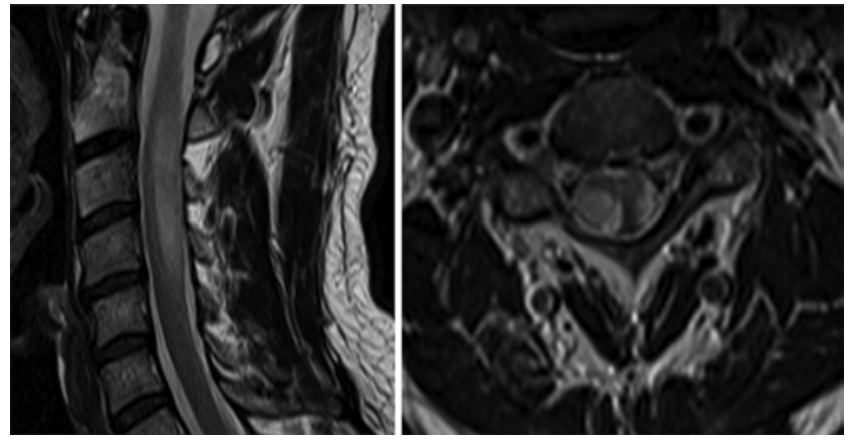

FIG. 3. T2 FLAIR sequence of an intramedullary metastasis of a known anaplastic astrocytoma WHO Grade III in the cervical spine. There was no enhancement in the T1-enhanced sequence. Sagittal T2 FLAIR sequence (left) and transversal MRI (right) are shown, with good visualization of the tumor.

croscope. Besides ultrasound, further methods used during surgery included the application of intravenous corticosteroids, $\mathrm{CO}_{2}$ laser, Cavitron ultrasonic surgical aspirator (CUSA), and intraoperative monitoring (IOM). Continuous intra- and postoperative administration of weightdependent intravenous corticosteroids over 24 hours was conducted in all cases, with the aim to diminish swelling of the spinal cord. The $\mathrm{CO}_{2}$ laser (Surgilase 25) was used 10 times (in $45 \%$ of patients). In 6 patients, ultrasound was additionally used during the tumor preparation to identify the borders to the healthy tissue. IOM was applied in $82 \%$ of patients. In 3 patients (14\%), a CUSA was used.

Postoperatively, 6 patients underwent radiation of the resected area with up to $30 \mathrm{~Gy}, 7$ had systemic chemotherapy, and 4 had a combination of these 2 treatments. In 2 cases, no further therapy was applied. In 1 case, a patient with a CUP did not want any further therapy, and the patient was discharged with no neurological symptoms. The source of the metastasis was still unknown 12 months after surgery, and the patient still did not show any neurological deficit.

Due to a general deterioration in 2 patients during the weeks after surgery, no further therapy was conducted in these cases. One patient with a kidney carcinoma treated with postsurgery radiation relapsed after 11 months at the same site and underwent a second surgery due to clinical worsening. A patient with a malignant melanoma showed a meningeal carcinomatous spreading in the follow-up MRI after 22 months and received a ventriculoperitoneal shunt 2 months later. Only 1 patient had a postoperative complication (a dural leakage), which had to be revised surgically.

One to 2 days after surgery, 11 of 19 patients (58\%) showed no neurological difference, with 4 patients $(21 \%)$ improving and 4 patients (21\%) worsening. Immediately after surgery, 11 patients $(58 \%)$ had either more or less trouble walking, compared with 7 patients $(37 \%)$ before surgery. Although the clinical outcome immediately after surgery showed a decrease in "walking safely" as determined by the mean McCormick Scale grade, there was no change in the Student's t-test $(\mathrm{p}=1.0)$.

For 5 patients who were treated before the year 1999, valid follow-up information could not be obtained. The last follow-up time for the patients still alive $(n=17)$ was a mean of 6.24 months (range 2-22 months) from the time of surgery. Death was confirmed in 11 patients, with a mean survival time of 11.6 months after surgery; the shortest survival time was 2 months and the longest was 30 months (data not shown in Table 3).

The clinical status represented by the McCormick Scale grade in the 17 patients for whom follow-up data were available showed a mean of 2.12, where 6 patients had almost no clinical disability (McCormick Scale Grade I) and 2 patients were severely disabled (McCormick Scale Grade IV). Compared with the clinical status 1-2 days after surgery, there was an improvement in the McCormick Scale grade at the last follow-up from 2.47 to $2.12(\mathrm{p}=0.009)$. Likewise, an improvement was detected when comparing the preoperative status $(2.45)$ with the last follow-up status $(2.12 ; \mathrm{p}=0.029)$.

\section{Discussion}

The occurrence of ISCM during the course of a malignant disease is still very rare. ${ }^{8,22,23,45,48,51}$ Nevertheless, with improved survival due to more effective treatments for many cancers, the incidence of ISCM will increase. This is already reflected by a continuous growth in the number of cases published in recent years (Fig. 4). Our findings support this trend, with almost $60 \%$ of all ISCM treated in the last 3 years out of a period of 22 years. There are a rising number of reports on the medical treatment of ISCM. However, generally applicable therapy algorithms are only just emerging. One explanation might be the considerable inconsistencies of published data in terms of localization, treatment option, and outcome of cancer patients with ISCM.

Comparing our series with other published reports, we find comparable data in terms of mean age at ISCM presentation (55 years) and delay of diagnosis. ${ }^{8,22,51}$ Interesting within this context is the fact that the delay in diagnosis of spinal pathologies shows a similar difference between men and women in spinal dural arteriovenous malformations, indicating that the often insidious onset of symptoms is frequently misinterpreted in women.

With respect to distribution between men and women, as well as localization of ISCM in the spinal cord, there is no clear picture emerging. In our series, $41 \%$ of ISCM were found in the cervical region, followed by the thoracic spine (36\%). Only $23 \%$ were located in the conus medullaris, which is in line with the findings of Kalayci et al..$^{22}$ but is in contrast to the results of Dam-Hieu et al., ${ }^{8}$ who describe the most common site of ISCM as in the conus medullaris $(11 / 19 ; 58 \%)$.

Our experience, with lung cancer being the primary tumor $(n=5,23 \%)$, followed by breast cancer $(n=3,14 \%)$, is shared with other publications. , $, 1,18,22,26,32,34,37,39,40,43,49$ The findings of systemic spreading are also similar to other published data. Only 1 patient showed $>1$ ISCM at the time of surgery. Nine patients (41\%) had no other metastatic manifestations, leaving more than $50 \%$ with systemic migration, of which almost $80 \%$ proved to have brain metastases. ${ }^{5,8,22,30}$ The metastatic spread of a malignant disease into the CNS, and especially into the spinal cord, is associated with a very poor prognosis, limiting the time of survival. $4,5,8,26,37,51$

Within our cohort, only 3 patients (14\%) had concom- 


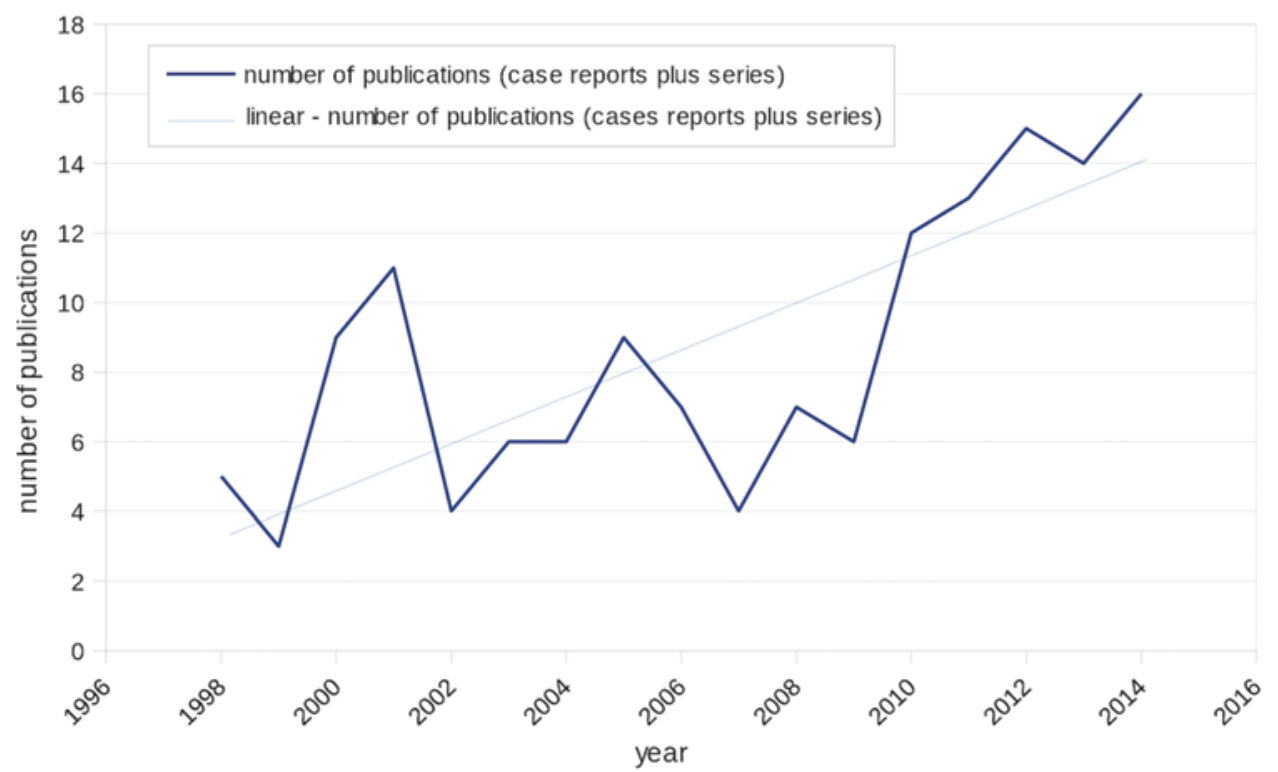

FIG. 4. Number of publications in PubMed including case reports, series of up to 19 cases, and review of the literature concerning intramedullary metastasis.

itant brain metastasis. One proved to be a metastasis of an unknown medulloblastoma in the cerebellum. Only very few cases of this entity are published. ${ }^{19,27}$ The second proved to be a metastasis originating from an ependymoma WHO Grade III, diagnosed in the brain 41 months prior to the spinal occurrence. Unfortunately, as these cases were treated in 1992 and 1999, respectively, the patients were lost to follow-up. The third brain metastasis originated from an anaplastic astrocytoma WHO Grade III, which was diagnosed 54 months earlier. ISCM was located in the cervical spine, and after biopsy in 2014, the patient was treated with radiation. The patient's neurological functions declined after biopsy (raising the McCormick Scale grade from II to III) and did not improve over time. However, the patient was still alive 6 months after surgery, with stable neurological impairment.

In 2 patients (9\%), a malignant melanoma proved to be the primary tumor, the percentage being similar to other published reports. ${ }^{17,33,51}$ The remaining histologies-small cell renal, bladder, prostate, and ovarian carcinomaswere represented by 1 case each. A review of the literature shows that these obviously rare types of ISCM are usually represented only by case reports. $1,2,7,9,13,14,16,21,28,35,36$

Interestingly, we encountered 5 cases (23\%) of ISCM as the primary finding of a malignant disease, which is very consistent with the findings of Kalayci et al..$^{22}$ but stands in contrast to those of Dam-Hieu et al. ${ }^{8}$ In the latter series, every patient except for 1 had a previously diagnosed tumor. ${ }^{8,22}$ In the case of 1 patient in our cohort, the origin of the primary tumor was still unknown 12 months after initial diagnosis. No second treatment after surgery has been conducted due to the decision of the patient and the absence of clinical impairment. This suggests that although ISCM are known to be a sign for poor prognosis, they still do not predict a fast lethal outcome in every case. Furthermore, length of survival apparently does not depend on the existence or absence of other metastatic manifesta- tions. One patient in our series presented with a neuroendocrine cancer without further systemic spreading but died 3 months after surgical intervention. Another patient, diagnosed with the same primary tumor and who also showed brain metastases at the time of surgery, died 30 months later. ISCM due to malignant melanoma as a primary cancer seems to be associated with a longer length of survival..$^{33}$ One patient developed a systemic meningeal carcinomatous after 22 months and received a ventriculoperitoneal shunt 2 months later. The last follow-up was after 25 months. The second patient with ISCM of malignant melanoma was treated in 2014 and is still alive. This finding is in line with the results of Wilson et al. ${ }^{51}$ as well as Conill et al. ${ }^{4}$

Using an ellipsoid volume assumption, taking into account peak open volume in width, length, and depth presented on the gadolinium-enhanced MRI before surgery, we could not find any correlation relating to length of survival, the McCormick Scale grade before and after surgery, tumor origin, or degree of resection. As there is no mention of any connection between size and any parameter in the literature, we cannot compare these findings with other authors' experiences.

Of the 18 patients (we were able to obtain up-to-date information from their family doctor), 11 died. In this group, the mean length of survival after spine surgery was 11.6 months. This is distinctly longer compared with reported survival times for many other series and meta-analyses. ${ }^{8,22,51}$ Comparing conservatively treated patients with surgically treated ISCM, Kalayci et al. demonstrated a surgical benefit in terms of increasing the length of survival from 5 months to 9.4 months, suggesting that surgical removal of the tumor without much delay has a positive effect on the further development of the disease. ${ }^{22}$ However, Wilson et al., ${ }^{51}$ as well as Watanabe et al., ${ }^{48}$ could not find a benefit from surgery concerning the length of survival in their respective series. Nevertheless, their patients had 
preoperative urinary retention, which is accompanied by a progressive neurological deficit and poor prognosis (in our series, only 5 cases).

Surgical resection of an ISCM should, if chosen as the primary therapy, aim for a total resection. Considering the importance of total tumor resection in the case of cerebral metastases, one could postulate a similar importance when surgically removing ISCM. On the one hand, similar to intracranial metastases, ISCM are often well delineated, allowing for a dissection plane such that gross total resection can be achieved with minimal parenchymal injury. On the other hand, as there is more edema in the parenchymal tissue than in the well-differentiated intrinsic intramedullary tumors, there is an increased vulnerability. Perioperative neuroprotection with high-dose methylprednisolone is used on a regular basis in combination with an IOM as feedback for possible surgical stress, so that surgical steps can be adapted and postsurgical impairment minimized. ${ }^{25,50}$ Intraoperative ultrasound is also used regularly to confirm adequate extent of exposure before opening the dura and to assess extent of tumor resection. ${ }^{12}$

$\mathrm{A} \mathrm{CO}_{2}$ laser is useful for minimizing mechanical stress during resection of an ISCM. It was applied in 10 cases in our series. Using all or a combination of these methods, it was possible to achieve at least a subtotal resection of the tumor in $85 \%$ of cases. Due to the heterogeneous origin of the primary histology, there is great variation in surface texture and infiltration at the tumor border. Therefore, in some cases, a small infiltrative tumorous layer was left deliberately. To enhance resection in these cases, a defocused, lowered-intensity $\mathrm{CO}_{2}$ laser coagulation after resection of the main tumor mass was performed. In the postoperative MRI, no contrast-enhanced tumor could be seen. In 7 patients (32\%), the surgeon described the anticipated total resection, which could be confirmed by postoperative MRI, showing no enhancement after gadolinium application. In 3 cases, only biopsy or partial removal was possible due to distinctive adhesions and worsening of intraoperative neuromonitoring.

Gasser et al. analyzed 146 spinal cord tumors, 13 of which were metastases, and postulated that radical resection depends on histology alone. Their data showed that sarcomas and poorly differentiated carcinomas could not be totally resected due to lack of a clear border between the tumor and the spinal cord. ${ }^{15}$ In our data, there was no correlation between the extent of resection and the postoperative clinical outcome or length of survival, which was also described by Gasser et al..$^{15}$

When extrapolating treatment of CNS metastases to the spinal cord, radiation therapy and chemotherapy have to be evaluated as part of treatment options. Radiation is usually considered for patients without neurological symptoms and can be well tolerated if the correct indications are made. ${ }^{8,31,38,44,47}$ On the other hand, Lee et al., ${ }^{26}$ in a series of 11 patients undergoing radiotherapy and 1 patient without treatment, and Conill et al. ${ }^{4}$ reporting on 6 cases treated with radiotherapy combined with steroids, showed that after a short period of clinical improvement, a rather fast decline occurred in neurological functions, sometimes within only a few days. Thus, one could assume that un- less radiation leads to an expected rapid shrinking of the tumor, surgery should be considered even when patients are oligosymptomatic.

Surgery is usually offered to patients suffering from neurological impairment or in the case of an unknown primary where a tissue diagnosis is required. ${ }^{30,44,47,51} \mathrm{Ka}-$ layci et al., having analyzed 32 published cases where surgery on ISCM was performed, showed that no patients developed new postoperative neurological deficits and an overall clinical improvement was found, with the length of survival being nearly twice as long compared with patients treated conservatively. ${ }^{22}$

Our study did not compare different modalities in the therapy of ISCM. Thus, we cannot answer the question of which therapy is better to use concerning postinterventional clinical decline or length of survival. It should be acknowledged that randomized trials to answer these clinical questions will probably never be performed due to recruitment barriers. But as can be seen throughout data published to date, each case must be considered individually to offer the best possible treatment. The different modalities involved-neurosurgery, radiotherapy, and oncology - must work closely together to achieve this. Surgery should be considered for the patient who shows fast neurological impairment, taking into consideration the primary disease (if known), the prognosis from the oncological point of view, and the decision of the patient. The preoperative neurological condition seems to be a reliable predictive factor for functional outcome after surgery and must therefore be taken into consideration when advising the patient. ${ }^{8,42,51}$ In the future, with an expected higher incidence of ISCM, initial neurological symptoms should be taken seriously and proper diagnostic measures initiated accordingly. Increasing neurological impairment seems to lead to a poor neurological outcome after surgery; thus, time is essential, the goal being to provide as much good quality of life as possible.

In cases of primary brain tumors or brain metastases, maximizing cytoreduction is known to have a positive effect on length of survival. This may also be essential in the case of ISCM and therefore should be set as the main goal of surgical treatment. ${ }^{24}$ Second, early decompression of the spinal cord may result in preserving or even improving neurological functions, as the growing understanding of spondylotic myelopathy has shown in the past. ${ }^{11}$ Taking all of this into account, the timely surgical treatment of ISCM must be considered an option to protect the remaining functional intact spinal cord. Technology for traction-free dissection, IOM, corticosteroids, potentially fluorescence guidance, and good anatomical knowledge help to achieve positive results. ${ }^{10}$

\section{Conclusions}

The occurrence of ISCM in the course of malignant disease is usually predictive of shortened life expectancy and is often associated with severe and worsening neurological deficits. We showed in 22 patients that surgery on ISCM, conducted under certain preconditions, can extend length of survival. The knowledge of incidence of intramedullary metastasis and its course is still very heterogeneous. 
It depends on the histology of the primary tumor, whether it has spread to other organs, operative and conservative treatment strategies, and the patient's overall condition. In the future, a sophisticated treatment algorithm will be of great importance, as the incidence of ISCM will probably increase further. Larger, prospective studies are needed.

\section{References}

1. Abdulazim A, Backhaus M, Stienen MN, Citak M, Brokinkel B, Kuhlmann T, et al: Intramedullary spinal cord metastasis and multiple brain metastases from urothelial carcinoma. J Clin Neurosci 18:1405-1407, 2011

2. Ateaque A, Martin JL, O'Brien C: Intramedullary spinal cord metastases from a hypernephroma 11 years following the diagnosis and treatment of the primary lesion. Br J Neurosurg 14:474-476, 2000

3. Chi JH, Parsa AT: Intramedullary spinal cord metastasis: clinical management and surgical considerations. Neurosurg Clin N Am 17:45-50, 2006

4. Conill C, Marruecos J, Verger E, Berenguer J, Lomeña F, Domingo-Domènech $\mathrm{J}$, et al: Clinical outcome in patients with intramedullary spinal cord metastases from lung cancer. Clin Transl Oncol 9:172-176, 2007

5. Connolly ES Jr, Winfree CJ, McCormick PC, Cruz M, Stein BM: Intramedullary spinal cord metastasis: report of three cases and review of the literature. Surg Neurol 46:329-338, 1996

6. Constantini S, Miller DC, Allen JC, Rorke LB, Freed D, Epstein FJ: Radical excision of intramedullary spinal cord tumors: surgical morbidity and long-term follow-up evaluation in 164 children and young adults. J Neurosurg 93 (2 Suppl):183-193, 2000

7. Crowley RW, Sherman JH, Le BH, Jane JA: Intramedullary spinal cord metastasis from bladder carcinoma: case report. Neurosurgery 63:E611-E612, 2008

8. Dam-Hieu P, Seizeur R, Mineo J-F, Metges J-P, Meriot P, Simon H: Retrospective study of 19 patients with intramedullary spinal cord metastasis. Clin Neurol Neurosurg 111:1017,2009

9. Donovan DJ, Freeman JH: Solitary intramedullary spinal cord tumor presenting as the initial manifestation of metastatic renal cell carcinoma: case report. Spine (Phila Pa 1976) 31:E460-E463, 2006

10. Eicker SO, Floeth FW, Kamp M, Steiger HJ, Hänggi D: The impact of fluorescence guidance on spinal intradural tumour surgery. Eur Spine J 22:1394-1401, 2013

11. Eicker SO, Langen KJ, Galldiks N, Stoffels G, Herdmann J, Steiger HJ, et al: Clinical value of 2 -deoxy-[ $\left.{ }^{18} \mathrm{~F}\right]$ fluoro-D-glucose positron emission tomography in patients with cervical spondylotic myelopathy. Neurosurg Focus 35(1):E2, 2013

12. Epstein FJ, Farmer JP, Schneider SJ: Intraoperative ultrasonography: an important surgical adjunct for intramedullary tumors. J Neurosurg 74:729-733, 1991

13. Fakih M, Schiff D, Erlich R, Logan TF: Intramedullary spinal cord metastasis (ISCM) in renal cell carcinoma: a series of six cases. Ann Oncol 12:1173-1177, 2001

14. Gao J, Li Y, Yang Z, Wang R: Intramedullary spinal cord metastasis of renal cell carcinoma 6 years following the nephrectomy. Turk Neurosurg 24:294-296, 2014

15. Gasser T, Sandalcioglu IE, El Hamalawi B, van de Nes JA, Stolke D, Wiedemayer H: Surgical treatment of intramedullary spinal cord metastases of systemic cancer: functional outcome and prognosis. J Neurooncol 73:163-168, 2005

16. Guo L, Zhong C, Jiang J, Qiu Y: A rare intramedullary spinal cord metastasis from prostate carcinoma. Neurol India 61:444-446, 2013
17. Hashii H, Mizumoto M, Kanemoto A, Harada H, Asakura $\mathrm{H}$, Hashimoto T, et al: Radiotherapy for patients with symptomatic intramedullary spinal cord metastasis. J Radiat Res (Tokyo) 52:641-645, 2011

18. Holoye P, Libnoch J, Cox J, Kun L, Byhardt R, Almagro U, et al: Spinal cord metastasis in small cell carcinoma of the lung. Int J Radiat Oncol Biol Phys 10:349-356, 1984

19. Inoue T, Kumabe T, Takahashi T, Nakajima T, Watanabe M, Tominaga T: Spinal intramedullary metastasis of medulloblastoma at initial diagnosis. Childs Nerv Syst 23:113-116, 2007

20. Ishii T, Terao T, Komine K, Abe T: Intramedullary spinal cord metastases of malignant melanoma: an autopsy case report and review of the literature. Clin Neuropathol 29:334-340, 2010

21. Isoya E, Saruhash Y, Katsuura A, Takahashi S, Matsusue Y, Hukuda S: Intramedullary spinal cord metastasis of ovarian tumor. Spinal Cord 42:485-487, 2004

22. Kalayci M, Cağavi F, Gül S, Yenidünya S, Açikgöz B: Intramedullary spinal cord metastases: diagnosis and treatment - an illustrated review. Acta Neurochir (Wien) 146:13471354,2004

23. Kalita O: Current insights into surgery for intramedullary spinal cord metastases: a literature review. Int J Surg Oncol 2011:989506, 2011

24. Kamp MA, Dibué M, Santacroce A, Zella SM, Niemann L, Steiger HJ, Rapp M, Sabel M: The tumour is not enough or is it? Problems and new concepts in the surgery of cerebral metastases. Ecancermedicalscience 7:306, 2013

25. Kothbauer K, Deletis V, Epstein FJ: Intraoperative spinal cord monitoring for intramedullary surgery: an essential adjunct. Pediatr Neurosurg 26:247-254, 1997

26. Lee SS, Kim MK, Sym SJ, Kim SW, Kim WK, Kim S-B, et al: Intramedullary spinal cord metastases: a single-institution experience. J Neurooncol 84:85-89, 2007

27. Madhugiri VS, Pandey P, Indira Devi B, Santosh V, Yasha TC: Intramedullary metastasis in a case of vermian medulloblastoma. Br J Neurosurg 26:278-280, 2012

28. Miranpuri AS, Rajpal S, Salamat MS, Kuo JS: Upper cervical intramedullary spinal metastasis of ovarian carcinoma: a case report and review of the literature. J Med Case Reports 5:311, 2011

29. Mortimer N, Hughes D, O'Byrne KJ: Intramedullary spinal cord metastasis. Lancet Oncol 2:607, 2001

30. Mut M, Schiff D, Shaffrey ME: Metastasis to nervous system: spinal epidural and intramedullary metastases. J Neurooncol 75:43-56, 2005

31. Nieder C, Grosu AL, Andratschke NH, Molls M: Update of human spinal cord reirradiation tolerance based on additional data from 38 patients. Int J Radiat Oncol Biol Phys 66:1446-1449, 2006

32. Nikolaou M, Koumpou M, Mylonakis N, Karabelis A, Pectasides D, Kosmas C: Intramedullary spinal cord metastases from atypical small cell lung cancer: a case report and literature review. Cancer Invest 24:46-49, 2006

33. Nishihara M, Sasayama T, Kondoh T, Tanaka K, Kohmura E, Kudo H: Long-term survival after surgical resection of primary spinal malignant melanoma. Neurol Med Chir (Tokyo) 49:546-548, 2009

34. Okamoto H, Shinkai T, Matsuno Y, Saijo N: Intradural parenchymal involvement in the spinal subarachnoid space associated with primary lung cancer. Cancer 72:2583-2588, 1993

35. Park J, Chung SW, Kim KT, Cho DC, Hwang JH, Sung JK, et al: Intramedullary spinal cord metastasis in renal cell carcinoma: a case report of the surgical experience. J Korean Med Sci 28:1253-1256, 2013

36. Poggi MM, Patronas N, Buttman JA, Hewitt SM, Fuller B: Intramedullary spinal cord metastasis from renal cell carci- 
noma: detection by positron emission tomography. Clin Nucl Med 26:837-839, 2001

37. Potti A, Abdel-Raheem M, Levitt R, Schell DA, Mehdi SA: Intramedullary spinal cord metastases (ISCM) and non-small cell lung carcinoma (NSCLC): clinical patterns, diagnosis and therapeutic considerations. Lung Cancer 31:319-323, 2001

38. Rades D, Rudat V, Veninga T, Stalpers LJA, Hoskin PJ, Schild SE: Prognostic factors for functional outcome and survival after reirradiation for in-field recurrences of metastatic spinal cord compression. Cancer 113:1090-1096, 2008

39. Rostami R, Safarpour D, Tavassoli FA, Jabbari B: Intramedullary metastasis in breast cancer-a comprehensive literature review. J Neurol Sci 332:16-20, 2013

40. Rykken JB, Diehn FE, Hunt CH, Schwartz KM, Eckel LJ, Wood CP, et al: Intramedullary spinal cord metastases: MRI and relevant clinical features from a 13-year institutional case series. AJNR Am J Neuroradiol 34:2043-2049, 2013

41. Samandouras G (ed): Oxford Medicine Online: The Neurosurgeon's Handbook. (http://www.oxfordmedicine. com/view/10.1093/med/9780198570677.001.0001/med9780198570677) [Accessed June 24, 2015]

42. Sandalcioglu IE, Gasser T, Asgari S, Lazorisak A, Engelhorn T, Egelhof T, et al: Functional outcome after surgical treatment of intramedullary spinal cord tumors: experience with 78 patients. Spinal Cord 43:34-41, 2005

43. Schiff D, O'Neill BP: Intramedullary spinal cord metastases: clinical features and treatment outcome. Neurology 47:906912, 1996

44. Shin DA, Huh R, Chung SS, Rock J, Ryu S: Stereotactic spine radiosurgery for intradural and intramedullary metastasis. Neurosurg Focus 27(6):E10, 2009

45. Sung WS, Sung MJ, Chan JH, Manion B, Song J, Dubey A, et al: Intramedullary spinal cord metastases: a 20-year institutional experience with a comprehensive literature review. World Neurosurg 79:576-584, 2013

46. Sutter B, Arthur A, Laurent J, Chadduck J, Friehs G, Clarici
G, et al: Treatment options and time course for intramedullary spinal cord metastasis. Report of three cases and review of the literature. Neurosurg Focus 4(5):e3, 1998

47. Veeravagu A, Lieberson RE, Mener A, Chen Y-R, Soltys SG, Gibbs IC, et al: CyberKnife stereotactic radiosurgery for the treatment of intramedullary spinal cord metastases. J Clin Neurosci 19:1273-1277, 2012

48. Watanabe M, Nomura T, Toh E, Sato M, Mochida J: Intramedullary spinal cord metastasis: a clinical and imaging study of seven patients. J Spinal Disord Tech 19:43-47, 2006

49. Weissman DE, Grossman SA: Simultaneous leptomeningeal and intramedullary spinal metastases in small cell lung carcinoma. Med Pediatr Oncol 14:54-56, 1986

50. Wiedemayer H, Fauser B, Sandalcioglu IE, Schäfer H, Stolke D: The impact of neurophysiological intraoperative monitoring on surgical decisions: a critical analysis of 423 cases. J Neurosurg 96:255-262, 2002

51. Wilson DA, Fusco DJ, Uschold TD, Spetzler RF, Chang SW: Survival and functional outcome after surgical resection of intramedullary spinal cord metastases. World Neurosurg 77:370-374, 2012

\section{Author Contributions}

Conception and design: Eicker, Payer. Acquisition of data: Eicker, Payer, Mende. Analysis and interpretation of data: Eicker, Payer. Drafting the article: Eicker. Critically revising the article: Mende, Westphal. Reviewed submitted version of manuscript: Westphal. Approved the final version of the manuscript on behalf of all authors: Eicker.

\section{Correspondence}

Sven O. Eicker, Department of Neurosurgery, University Hospital Hamburg-Eppendorf, Martinistr. 52, Hamburg 20246, Germany. email: eicker.s@mac.com. 